\section{The London Depression Intervention Trial}

\author{
Randomised controlled trial of antidepressants $v$. couple
}

therapy in the treatment and maintenance of people with

depression living with a partner: clinical outcome and costs ${ }^{\dagger}$

J. LEFF, S. VEARNALS, C. R. BREWIN, G. WOLFF, B. ALEXANDER, E. ASEN, D. DAYSON, E. JONES, D. CHISHOLM and B. EVERITT

\author{
Background Relapse of depression is \\ associated with a criticising attitude of the \\ patient's partner.
}

\begin{abstract}
Aims To compare the relative efficacy and cost of couple therapy and antidepressant drugs for the treatment and maintenance of people with depression living with a critical partner.
\end{abstract}

Method A randomised controlled trial of antidepressant drugs $v$. couple therapy. The subjects were 77 people meeting criteria for depression living with a critical partner.

Results Drop-outs were $56.8 \%$ from drug treatment and $15 \%$ from couple therapy. Subjects' depression improved in both groups, but couple therapy showed a significant advantage, according to the Beck Depression Inventory, both at the end of treatment and after a second year off treatment. Adding the costs of the interventions to the costs of services used showed there was no appreciable difference between the two treatments.

Conclusions For this group couple therapy is much more acceptable than antidepressant drugs and is at least as efficacious, if not more so, both in the treatment and maintenance phases. It is no more expensive overall.

Declaration of interest Funding from the Medical Research Council.

†See editorial, pp. 93-94, this issue.
A number of studies of patients living with partners have shown that the outcome of depressive neurosis over 9 months is associated with the degree of criticism expressed by the partner towards the patient (Vaughn \& Leff, 1976b; Hooley et al, 1986; Okasha et al, 1994), although one recent study failed to replicate this finding (Hayhurst et al, 1997). This association suggests that couple therapy might be an appropriate form of treatment for patients with depression living with a critical partner. A small number of randomised controlled trials of marital therapy for such patients have been published (Friedman, 1975; Waring et al, 1988; O'Leary \& Beach, 1990; Jacobson et al, 1991; Emanuels-Zuurveen \& Emmelkamp, 1996). The duration of these trials varied between 10 and 20 weeks and none included a follow-up period after treatment to assess any prophylactic effect. There was a consistent finding that marital therapy and cognitive therapy were equally effective in reducing depressive symptoms, but that only marital therapy improved the marital relationship.

\section{METHOD}

The intention was to compare the optimum regime of antidepressants with couple therapy delivered by experienced therapists. Antidepressant treatment would be maintained for a year, after which it would be discontinued. Couple therapy would be given as an alternative to drugs during the first year. The second year of the trial would represent the prophylactic phase for both treatments, which would end with a follow-up assessment. A comparison of the costs of treatment and other services received was included, in order to compare the cost-effectiveness of the two treatments.

\section{Subjects}

Subjects were recruited in different ways. The majority came through professional contacts, including general practitioners in north and south London, and the Maudsley Hospital out-patient service and emergency clinic. A minority contacted us in response to advertisements in local newspapers. Subjects had to be under the age of 65 and had to have lived with a heterosexual partner for at least 1 year. They also needed to reach a level of 'caseness' (Index of Definition level 5 or above), receive a primary classification of depression on the Present State Examination - Catego System (Wing et al, 1974) and achieve a score on the Hamilton Depression Rating Scale (Hamilton, 1960) of at least 14. These criteria define a major depressive episode. The partner had to be rated as expressing at least two critical comments during the Camberwell Family Interview (Vaughn \& Leff, 1976a).

Patients were excluded if they showed any psychotic features, or suffered from bipolar illness, organic brain syndrome, severe suicidal tendencies, primary substance abuse, learning difficulties, or contraindications (such as pregnancy) for antidepressants. Those who had during the preceding 3 months experienced an adequate course of either of the treatments offered in the trial were also excluded. The criteria for adequacy were 6 weeks of an antidepressant at a therapeutic dosage, or six sessions of couple therapy.

A power calculation based on the relapse rates in the naturalistic studies of critical partners and the course of depression indicated that for a significance level of $P<0.05$ and a power of $80 \%$, a sample size of 40 in each group was required. The research team made contact with 290 individuals, of whom 196 were excluded because they did not have a stable relationship or would not accept random allocation to treatment. Of the remaining 94 people, six rejected the possibility of receiving antidepressants. This left 88 subjects and their partners who met our criteria, who were randomised to one of the trial groups, using a computer-generated random number table and sealed envelopes. As part of the randomisation procedure, subjects were stratified according to whether or not they had a significant history of depression, defined as a current episode of depression lasting 6 months or more or a previous treated episode in the last 3 years. 


\section{Treatments}

Initially we had intended to compare three treatments for depression: antidepressants, couple therapy and cognitive therapy. We anticipated recruiting three subjects per month, but the rate actually achieved was two per month, and the drop-out rate from the cognitive therapy arm was so high $(8$ out of 11) that this treatment option was soon deleted from the trial. Hence we present here the results for the 77 subjects who received antidepressants or couple therapy. We did not include a group of subjects who were assigned to no treatment, as this was considered unethical, given the well-established efficacy of antidepressants for major depressive episodes.

\section{Antidepressant regime}

The pharmacotherapy was the responsibility of D.D., who selected a regime to represent the best available clinical practice, while ensuring compliance. The initial medication was a tricyclic antidepressant, desipramine, provided there was no contraindication. The dose was gradually increased over a few weeks, titrating it against side-effects and symptom reduction. To monitor compliance independently of the patient's report, serum levels were checked at 4 weeks, 8 weeks, 6 months and 1 year. If, despite taking desipramine for a full 6 weeks in doses that achieved therapeutic serum levels $(125 \mu \mathrm{g} /$ $\mathrm{ml}$ ), there was no response, or intolerable side-effects developed, a second-line antidepressant was substituted: if agitation was prominent, trazodone was prescribed, whereas if the patient showed retardation, fluvoxamine was given. Once an effective dose had been achieved and symptoms remitted, the patient was continued on this dose for 4 months, following which the dose was gradually reduced to between $1 /$ 2 and 2/3 of the peak dose. Patients were maintained on this dose for the rest of the first year. Alongside medication, during the first two sessions an educational programme about depression and antidepressants was given to the patient and their partner, to maximise compliance. The sessions lasted 20-30 minutes, those who completed the course received between 12 and 20 sessions. After 1 year the antidepressant was tailed off over 2 weeks, although two patients chose to remain on medication, and two others relapsed as soon as it was stopped, necessitating a resumption.

\section{Couple therapy}

Two senior family and couple therapists were responsible for developing the form of treatment used in this trial. In their model the patients' depressive symptoms are conceptualised in interactional terms. Close relationships are regarded both as influencing, and being influenced by, the patient and his/her symptoms. Systemic couple therapy aims to help the patient and partner to gain new perspectives on the presenting problems, to attach different meanings to the depressive types of behaviour and to experiment with new ways of relating to each other. During a pilot phase, the therapists constructed a manual, and modified it through experience with six couples who met the criteria for the trial but were not randomly allocated to treatment. During this phase the therapists attempted to design specific interventions aimed at directly reducing criticism by the partner. However, the techniques used seemed to have little effect on the patient's depressive symptoms and were therefore not included in the final version of the protocol.

The manual (Jones \& Asen, 1999) specifies in detail the techniques to be employed, such as observation and enactment of couple issues, attempts to interrupt problematic cycles of behaviour to shift negative attributions, and setting of tasks to develop less problematic ways of interacting. The manual describes three distinct phases of therapy in which various specific interventions are used, while allowing for enough flexibility to avoid the therapy becoming a strait-jacket for everyone concerned. The protocol allowed for 12-20 sessions, lasting around 50 minutes each.

\section{Assessments}

The initial assessment of the patient included a full psychiatric history, the Present State Examination (PSE), the Hamilton Rating Scale for Depression (HRSD), and the Beck Depression Inventory (BDI) (Beck et al, 1961). The partner was assessed by means of the Camberwell Family Interview (CFI), while both patient and partner completed the Dyadic Adjustment Scale (Spanier, 1976) to measure the quality of their relationship. Following the completion of treatment, and at the end of a second year without treatment, these assessments were repeated for patient and partner by researchers who were blind to the treatment group of the subjects.
Changes in the CFI and the Dyadic Adjustment Scale will be presented in another paper dealing with the mechanisms of action of the treatments.

\section{Economic analysis}

The cost of couple therapy was calculated on the basis of the number (and average duration) of sessions and the unit cost per hour of direct research therapist's contact time. All prescribed antidepressants (and associated blood tests) over the course of the study were recorded and costed, so that drug therapy costs could be estimated. Service utilisation data were collected using a variant of the Client Service Receipt Inventory (CSRI; Beecham \& Knapp, 1992), which covered a range of key health and social care services (in-, out-, and day patient hospital services, day care and contacts with a general practitioner, community psychiatric nurse, social worker and counsellor). Unit costs were attached to these data, and aggregated to give a total service cost estimate for each subject in the study. Unit cost figures were calculated to represent long-run marginal opportunity costs, and were drawn from national estimates, adjusted for London as necessary (Netten \& Dennett, 1996). Informal caregiver support by family members or others, and the indirect consequences of depression (lost employment), were not costed in this study. Analysis of cost differences between the two groups focused on the mean (rather than the median) as the measure of direct policy interest, using a non-parametric bootstrap for the estimation of $95 \%$ confidence intervals, owing to the positively skewed distribution observed for service cost variables (Barber \& Thompson, 1998).

\section{Statistical analysis}

Each outcome variable provided data at three time points: baseline, 1-year and 2-year follow-up. Various methods of analysis have been proposed for such longitudinal data (Everitt, 1985, 1998; Diggle et al, 1994). Because of the loss of data when patients dropped out, it was decided to use the likelihood approach, originally described by Schluchter (1988) and implemented in the BMDP statistical package as BMPD5V. This method allows for drop-outs, and produces valid parameter estimates and standard errors as long as the drop-outs are not informative (Everitt, 1998). Even when the drop-outs are informative, the likelihood method will 


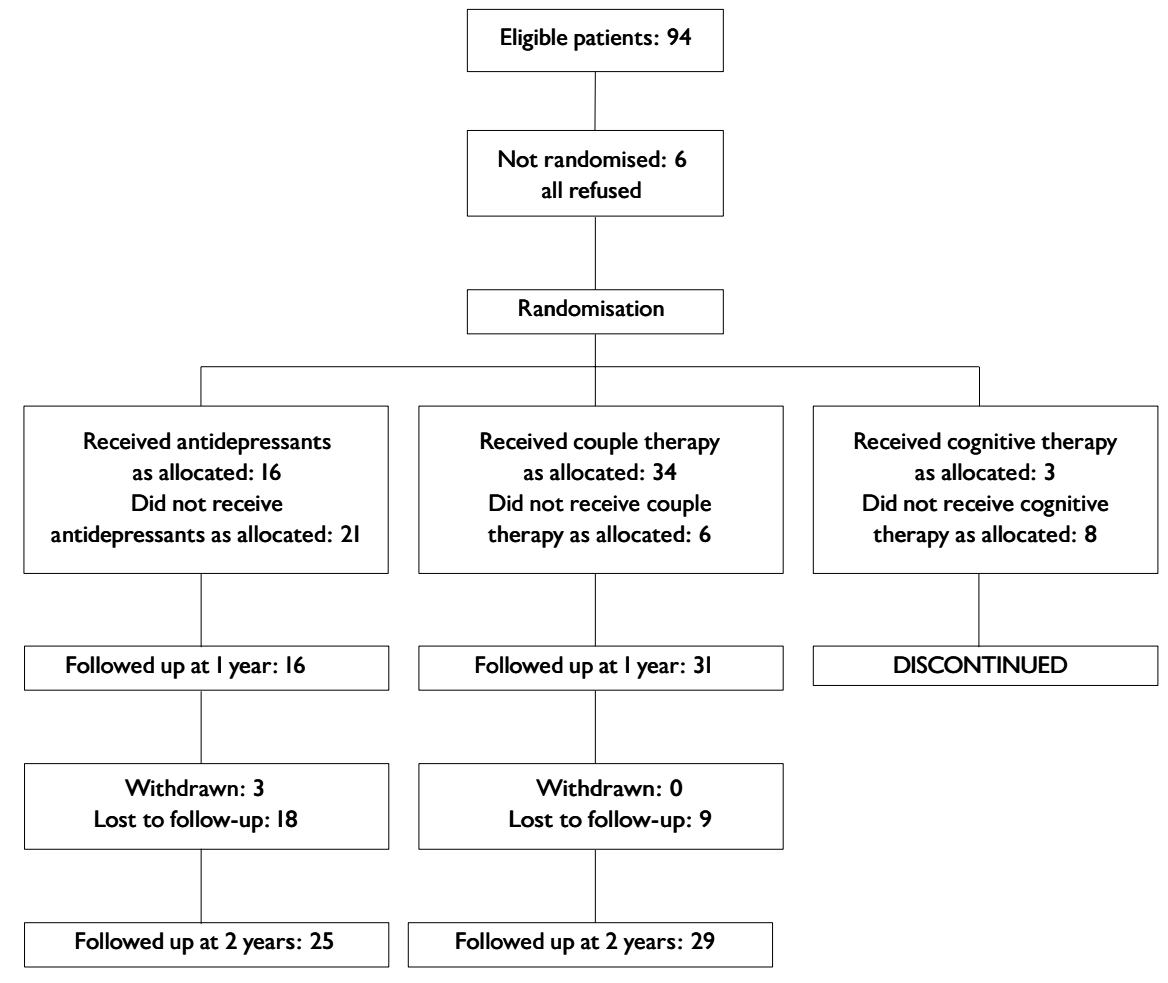

Fig. I CONSORT diagram.

produce a less biased analysis than commonly used alternatives, such as analysing only those who complete the course, or replacing missing values with the last available measurement (last observation carried forward) (Diggle, 1998). The analysis included all patients for whom data were available for at least one of the 1-year and 2-year follow-ups in addition to the baseline assessment. The 1-year follow-up sample comprised only those who had completed the treatment, while the 2-year follow-up sample also included some patients who had dropped out of treatment prematurely.

We used the BDI and the HRSD as indicators of outcome but excluded the PSE, as it is primarily a diagnostic instrument and is not sensitive to incremental changes over time.

\section{RESULTS}

\section{Subjects}

Of the 94 patients assessed as suitable for the trial over 45 months, only six refused to enter the study. This is an unusually low refusal rate, but we have to take into account the large number of patients with whom we made contact who refused to be assessed. As we have no data on these people, we cannot compare them with the trial subjects. A further 11 were lost when the cognitive therapy treatment was deleted early in the trial. Of the 77 patients in the two-arm trial, just under one quarter referred themselves in response to advertisements. The characteristics of the patients assigned to the two therapies are shown in Table 1.

Table I Characteristics of subjects in the two treatment groups

\begin{tabular}{lll}
\hline & $\begin{array}{c}\text { Drug treatment } \\
n=37\end{array}$ & $\begin{array}{c}\text { Couple therapy } \\
n=40\end{array}$ \\
\hline Patient's mean age, years (s.d.) & $38.6(9.2)$ & $39.7(12.5)$ \\
Partner's mean age, years (s.d.) & $39.1(9.8)$ & $40.9(15.0)$ \\
Gender of patient: M/F & $10 / 27$ & $17 / 23$ \\
Duration of current relationship, years (s.d.) & $10.1(7.8)$ & $10.8(11.8)$ \\
$\quad$ Median: percentile (25, 75) & $7.0(3.5,15.0)$ & $5.0(3.0,17.25)$ \\
Dyadic Adjustment Scale (initial) (s.d.) & $96.5(20.1)$ & $87.3(24.7)$ \\
Partner's critical comments (initial) (s.d.) & $8.8(9.8)$ & $8.3(6.4)$ \\
Age of onset of first depression, years (s.d.) & $28.6(9.3)$ & $29.8(16.1)$ \\
$\quad$ Median: percentile (25, 75) & $28.0(21.0,34.5)$ & $25.0(16.0,39.75)$ \\
Significant history of depression & 30 & 30 \\
Hamilton Depression Rating Scale (initial) (s.d.) & $18.7(3.9)$ & $18.1(3.4)$ \\
Beck Depression Inventory (initial) (s.d.) & $28.1(6.0)$ & $25.4(7.4)$ \\
Source of referral (\%) & & 75.0 \\
$\quad$ Professional & 75.7 & 25.0 \\
Advertisement & 24.3 & \\
\hline
\end{tabular}

Patients' progress through the trial is shown in a CONSORT diagram (see Fig. 1).

\section{Drop-outs}

Of the 37 patients randomly allocated to the drug treatment, nine dropped out before their first treatment visit, and another 12 subsequently. Three of these patients were withdrawn from the trial by the pharmacotherapist, two because they developed mania (an exclusion category for the trial) and one on account of intolerable side-effects. Only two people objected to having blood tests: one had severe needle phobia and the other refused tests, but both continued with the treatment. All the tests showed that patients had antidepressant levels within the therapeutic range. Of the 40 patients randomly allocated to couple therapy, three dropped out before any treatment, and a further three subsequently. The difference in total drop-out rate between drug treatment $(56.8 \%)$ and couple therapy $(15.0 \%)$ is $41.8 \% \quad(95 \%$ CI 31.8-51.8) and is highly significant $\left(\chi^{2}=14.72\right.$, d.f. $\left.=1, P<0.001\right)$.

Drop-outs were compared with those who completed treatment as regards several variables, in order to identify what might determine failure to complete treatment. No differences were found between the two groups regarding gender, Dyadic Adjustment Score, partner's critical comments or HRSD score. However, the drop-outs were significantly younger than 


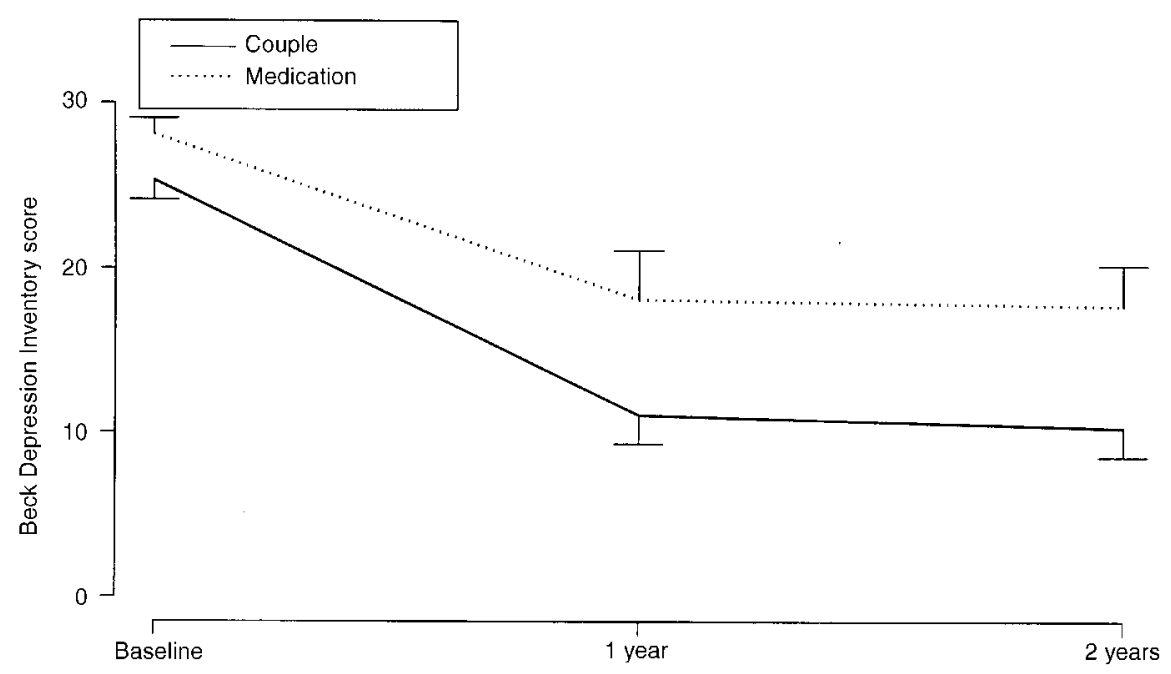

Fig. 2 Mean profiles of couple and medical treatment groups.

the completers $(34.3 v .41 .8, \quad t=3.30$, d.f. $=75, P<0.002)$, and had higher BDI scores $(29.4 v$. 25.2, $t=2.99$, d.f. $=75$, $P<0.004)$. The assessments at the 1 -year follow-up were only conducted on subjects who completed treatment. However, at the 2-year follow-up, a small financial inducement persuaded a number of dropouts, particularly those in the drug group, to return for assessment.

\section{Effects of treatment}

The statistical analysis was conducted on an intention-to-treat basis, and any data available from drop-outs were included. The likelihood approach was used to fit a variety of models for the mean profiles of the BDI outcome variable. The main effects of treatment group and time, and the interaction of treatment group and time, were considered. In addition, the initial value of the BDI was introduced as a covariate, along with age and a small number of additional covariates such as gender and history of depression. Only treatment group was found to be significant, with an average difference in BDI scores of 6.4 (95\% CI 1.62-11.54). In the absence of a significant group $\times$ time interaction, this implies that from initial assessment to the 1-year follow-up, the mean BDI score in the couple therapy group falls to between 1.62 and 11.54 points lower than the corresponding mean in the medication group. This difference is maintained from the 1-year time point until the 2-year follow-up. Figure 2 shows the mean scores and standard errors of the means over the three time points.

The same analysis was applied to the HRSD scores, and produced a different result, namely that a significant improvement had occurred after treatment for both groups, which was sustained during the year without treatment, but that there was no significant advantage for couple therapy over medication.

\section{Economic component}

The collection of data regarding use of services, necessary for the economic evaluation of the alternative therapy strategies, did not form part of the original battery of instruments, so that these data were missing for initial recruits to the trial $(27$ cases). Drop-outs during the treatment phase who failed to return for the 2-year follow-up, loss of contact over the followup phase with those who had completed treatment, and non-consent at both assessment points also contributed to a considerable reduction in the sample available for economic analysis.

Table 2 gives the costs of therapy and service use over the treatment and followup phases of the study. It should be noted that the drug treatment costs include the cost of the blood tests (a mean of $£ 3.73$ per month), which would not be done routinely. The mean monthly cost of couple therapy was $£ 106$ (s.d.=23), compared with a treatment cost of $£ 48$ (s.d. $=18$ ) for the drug group. This significantly greater cost of treatment $(£ 58,95 \%$ CI $45-72)$ is offset by a reduction in the costs of hospital

Table 2 The costs of therapy and service utilisation

\begin{tabular}{|c|c|c|c|c|c|c|c|c|c|c|}
\hline \multirow{2}{*}{$\begin{array}{l}\text { Cost component } \\
(£ \text { per month, 1995-1996) }\end{array}$} & \multicolumn{4}{|c|}{ Couple therapy group } & \multicolumn{4}{|c|}{ Drug therapy group } & \multicolumn{2}{|c|}{ Mean difference } \\
\hline & $n$ & Median & Mean & s.d. & $n$ & Median & Mean & s.d. & Mean & $95 \% \mathrm{Cl}^{2}$ \\
\hline \multicolumn{11}{|l|}{ Treatment period } \\
\hline A. Therapy & 24 & 108 & 106 & 23 & 14 & 46 & 48 & 18 & +58 & 45 to 72 \\
\hline B. Hospital and community services & 24 & 12 & 25 & 36 & 14 & 14 & 78 & 134 & -53 & -130 to 10 \\
\hline Hospital services & & I & 17 & 35 & & I & 38 & 104 & -20 & -87 to 20 \\
\hline Community services & & 5 & 8 & 9 & & 7 & $4 I$ & 95 & -32 & -91 to 1.4 \\
\hline C. Combined cost $(A+B)$ & 24 & 132 & $|3|$ & 38 & 14 & 62 & 126 & 139 & +5 & -76 to 71 \\
\hline \multicolumn{11}{|l|}{ Follow-up period' } \\
\hline D. Hospital and community services & 27 & 16 & 43 & 60 & 21 & 16 & 67 & 122 & -24 & -83 to 25 \\
\hline Hospital services & & 10 & 29 & 42 & & 7 & 56 & $|2|$ & -26 & -88 to 19 \\
\hline Community services & & 2 & 14 & 48 & & 8 & 12 & 12 & +2 & -9 to 20 \\
\hline
\end{tabular}

I. Includes assessment of people who dropped out of treatment.

2. $95 \%$ confidence intervals obtained by non-parametric bootstrapping (1000 repetitions). 
and community services (a mean of $£ 53$ per month less in the couple therapy group, $95 \%$ CI -130 to 10$)$. As evidenced by the low median values and large standard deviations, however, these service costs varied markedly, and mean differences were not statistically significant. The combined mean of therapy and service use is similar for the two groups $(£ 5$ higher in the couple therapy group; $95 \%$ CI -76 to 71 ).

The costs of service use were also monitored over the follow-up period of the study, and included costs incurred by treatment-phase drop-outs who agreed to be interviewed. Mean service costs were higher in the drug group ( $£ 67$, s.d. 122) than in the couple therapy group ( $£ 43$, s.d. 60), but this difference was not statistically significant ( $£ 24,95 \% \mathrm{CI}-83$ to 25$)$, again due to the skewed nature of the data (median costs per month for both groups were only $£ 16$ ). It is noticeable (but not tabulated here) that the cost of hospital service use over the follow-up period for the ten cases who did not engage in either form of therapy ( $£ 111$, s.d. 170$)$ was three times as great as that for the 38 cases who did engage in therapy ( $£ 38$, s.d. 52).

\section{DISCUSSION}

\section{Reasons for dropping out}

More than half the subjects dropped out of drug treatment, compared with $15 \%$ of those having couple therapy. This finding in itself shows that drug treatment was far less acceptable to the patients in the study than was couple therapy, providing further evidence for the popular bias against antidepressants which has been documented by other studies (Scott \& Freeman, 1992; Whitton et al, 1996). Blood testing did not lead to dropping out, and the tests showed that those who continued with medication were fully compliant, an achievement which must be partly attributable to the education programme given to patients and their partners. The drop-out from the drug arm appears high in relation to other trials of antidepressants (Hollyman et al, 1988; Elkin et al, 1989), but few have continued for as long as 1 year. This duration of treatment was recommended by the expert we consulted, and seems appropriate, given that three-quarters of the patients in the trial had been depressed for at least 6 months or had had a treated episode in the previous 3 years. Patients who dropped out of our trial were younger and more depressed initially than those who completed it. However, the inclusion of these factors as covariates in the statistical analysis accommodates to some extent for any bias introduced.

\section{Differences between the outcome assessments}

The analysis demonstrated the superiority of couple therapy over antidepressant drugs, both for the treatment phase and for the maintenance phase, as judged by the BDI scores. By contrast, the HRSD scores did not discriminate between the improvements in the two treatment groups. This may be because the two scales have a different proportion of cognitive-affective items: $67 \%$ of the BDI and $29 \%$ of the HRSD. Furthermore, the BDI has 4-point scales for each item, whereas the HRSD requires yes/no replies. Senra \& Polaino (1998) consider that discrepancies between the HRSD and BDI as regards the degree of improvement brought about by treatment are essentially due to differences in scale content. Comparison with previous controlled trials of marital therapy is not easy, since in three of the five studies the other treatment was cognitive therapy, a modality which was dropped from our trial. Of the other two studies, Waring et al (1988) included too few subjects in each cell to draw valid conclusions. The numbers were reasonably large in the trial by Friedman (1975) but that continued for only 12 weeks. It was a four-cell design, with random assignment to amitriptyline or placebo, and to weekly marital therapy or to minimal contact. Neither amitriptyline nor marital therapy showed any advantage over the control treatment in alleviating depression. However, patients who received marital therapy rated the marriage as better at follow-up than those in the minimal contact group. In the absence of any directly comparable study, our finding of an apparent advantage of couple therapy over antidepressants needs to be replicated in further trials before being accepted as proven.

\section{Comparative costs of the treatments}

The addition of a costing component to the study illustrated that the cost of couple therapy was approximately double that of a conventional antidepressants. The observed difference in treatment cost appears to be moderated by a reduced use of other services. What distinguishes the two groups more than any difference in total direct cost is the constitution of the costs (couple therapy: $81 \%$ treatment, $19 \%$ service use; drug group: $38 \%$ treatment, $62 \%$ service use). Alongside the improvement in BDI scores observed for the couple therapy, this provides encouraging evidence for the cost-effectiveness of this intervention. However, the post-design addition of the service utilisation schedule, plus subsequent non-completion and drop-outs, reduced the size of the sample for whom costs could be estimated to a small one only. Taken in conjunction with the highly positively skewed distribution of service costs, this leaves us much less capable of commenting on the findings with any statistical confidence. Also, only direct costs of care and support were included: the wider economic consequences of depression, including the costs associated with work disability, were not fully considered.

\section{Are the results generalisable?}

To what extent can these findings be applied to the general population of patients with depression? Clearly they cannot be extrapolated beyond those living with a heterosexual partner. One selection criterion for our sample was that the partner had to make more than one critical comment on the CFI. In fact, only a handful of patients was excluded on this basis. Not only do patients with depression living with a partner greatly prefer couple therapy to antidepressant drugs: it is also at least as efficacious, and may be superior, both in the treatment and the maintenance phases. The economic analysis has to be treated with caution because of missing data, but does suggest that the higher cost of couple therapy is compensated for by less expenditure on other services used by the patients. We consider that our findings constitute a strong argument for training primary care personnel in the skills of couple therapy.

\section{ACKNOWLEDGEMENT}

We are grateful to Mrs Yvonne Waters for her efforts in maintaining contact with the subjects over the 2-year follow-up.

\section{REFERENCES}

Barber, J. A. \& Thompson, S. G. (1998) Analysis and interpretation of cost data in randomised controlled 
trials: review of published studies. British Medical Journal, 317, $1195-1200$.

Beck, A. T., Ward, C. H., Mendelson, M., et al (1961) An inventory for measuring depression. Archives of General Psychiatry, 4, 56I-57I.

Beecham, J. K. \& Knapp, M. R. J. (1992) Costing psychiatric interventions. In Measuring Mental Health Needs (eds G. Thornicroft, C. R. Brewin \& J. K.Wing) London: Gaskell.

Diggle, P. J. (1998) Dealing with missing values in longitudinal studies. In Statistical Analysis of Medical Dato (eds B. S. Everitt \& G. Dunn). London: Arnold.

\section{—, Liang, K. \& Zeger, S. L. (1994) Analysis of Longitudinal Data. Oxford: Oxford University Press.}

Elkin, I., Shea, M.T., Watkins, J.T., et al (1989)

National Institute of Mental Health Treatment of Depression Collaborative Research Program. General effectiveness of treatments. Archives of Generol Psychiatry, 46, 971-982.

Emanuels-Zuurveen, L. \& Emmelkamp, P. M. G. (1996) Individual behavioural-cognitive therapy $v$. marital therapy for depression in maritally distressed couples. British Journal of Psychiatry, 169, 181-188.

Everitt, B. S. (1985) The analysis of repeated measures: a practical review with examples. Statistician, $\mathbf{4 4}$ 113-135.

— (1998) Analysis of longitudinal data. Beyond MANOVA. British Journal of Psychiatry, 172, 7-10.

Friedman, A. S. (1975) Interaction of drug therapy with marital therapy in depressive patients. Archives of General Psychiatry, 32, 619-637.

Hamilton, M. (1960) A rating scale for depression Journal of Neurology, Neurosurgery and Psychiatry, $\mathbf{2 3}$ $59-61$

Hayhurst, H., Cooper, Z., Paykel, E. S., et al (1997) Expressed emotion and depression. A longitudinal study. British Journal of Psychiatry, I7I, 439-443.

Hollyman, J. A., Freeling, P., Paykel, E. S., et al (1988) Double-blind, placebo-controlled trial of amitriptyline among depressed patients in general practice. Journal of the Royal College of General Practitioners, 38, 393-397.

Hooley, J. M., Orley, J. \& Teasdale, J. D. (1986) Level of expressed emotion and relapse in depressed patients. British Journal of Psychiatry, 148, 642-647.

Jacobson, N. S., Dobson, K., Fruzzetti, A. E., et a (1991) Marital therapy as a treatment for depression. Journal of Consulting \& Clinical Psychology, 59, 547-557.

Jones, E. \& Asen, E. (1999) Systemic Couple Therapy and Depression. London: Karnac

Netten, A. \& Dennett, J. (1996) Unit Costs of Health and Social Care Canterbury: Personal Social Services Research Unit, University of Kent.

Okasha, A., El Akabawi, A. S., Snyder, A. S., et al (1994) Expressed emotion, perceived criticism, and relapse in depression: a replication in an Egyptian community. American Journal of Psychiatry, I5I |00|-1005.

O'Leary, K. D. \& Beach, S. R. H. (1990) Marital therapy. A viable treatment for depression and marital discord. American Journal of Psychiatry, 147, $183-186$

\section{CLINICAL IMPLICATIONS}

Patients with depression living with a critical partner find couple therapy much more acceptable than antidepressant drugs.

- Couple therapy is at least as efficacious as antidepressant drugs, if not more so, both as a treatment for depression and in maintaining patients free of symptoms subsequently.

- Combining treatment costs with the costs of other services used, couple therapy is no more expensive than medication.

\section{LIMITATIONS}

Recruitment of subjects was from a wide variety of sources, so that the findings cannot readily be extrapolated to a typical general practice.

- The high drop-out rate from the medication group made statistical comparison more difficult, but was partly accommodated by the method of analysis.

- The costing of service use did not start at the initiation of the study, leading to a small sample size for this analysis. Furthermore, indirect costs were not assessed.

JULIAN LEFF, FRCPsych, SIMON VEARNALS, BSc, GEOFFREY WOLFF, MD, BARBARA ALEXANDER, DPsych, Social Psychiatry Section; DANIELCHISHOLM, MA, Centre for the Economics of Mental Health, BRIAN EVERITT, PhD, Department of Biostatistics \& Computing, Institute of Psychiatry, London; EIA ASEN, FRCPsych, Psychotherapy Department, Bethlem \& Maudsley NHS Trust, London; ELSA JONES, MA, The Family Institute, Cardiff; CHRIS R. BREWIN, PhD, Department of Psychology, Royal Holloway College, Egham; DAVID DAYSON, MRCPsych, Royal South Hants Hospital, Southampton

Correspondence: Professor Julian Leff, Head of Social Psychiatry Section, Institute of Psychiatry De Crespigny Park, Denmark Hill, London SE5 8AF

(First received I3 November 1998, final revision 4 February 2000, accepted I0 February 2000)

Schluchter, M. D. (1988) Analysis of incomplete multivariate data using linear models with structured covariance matrices. Statistics in Medicine, $\mathbf{7}$ 317-324.

Scott, A. I. F. \& Freeman, C. P. L. (1992) Edinburgh primary care depression study: treatment outcome patients' satisfaction, and cost after 16 weeks. British Medical Journal, 304, 883-887.

\section{Senra, C. \& Polaino, A. (1998) Assessment of} treatment outcome in depressed patients. Concordance of methods. British Journal of Clinical Psychology, 37, 217-227.

Spanier, G. B. (1976) Measuring dyadic adjustment: new scales for assessing the quality of marriage and similar dyads. Journal of Marriage \& the Family, 38, 15-28.

Vaughn, C. \& Leff, J. P. (1976a) The measurement of expressed emotion in families of psychiatric patients. British Journal of Social \& Clinical Psychology, 15 I57-165.
_ \& _ (1976b) The influence of family and socia factors on the course of psychiatric illness. A comparison of schizophrenic and depressed neurotic patients. British Journal of Psychiatry, 129, 125-137.

Waring, G. M., Chamberlaine, C. H., McCrank, E.W., et al (1988) Dysthymia: a randomized study of cognitive marital therapy and antidepressants. Canadian Journal of Psychiatry, 33, 96-99.

Whitton, A., Warner, R. \& Appleby, L. (1996) The pathway to care in post-natal depression: women's attitudes to post-natal depression and its treatment. British Journal of General Practice, 46 427-428.

Wing, J. K., Cooper, J. E. \& Sartorius, N. (1974) The Measurement and Classification of Psychiatric Symptoms. Cambridge: Cambridge University 